\title{
PROTOTYPE BUNCH KILLER SYSTEM AT SRRC
}

\author{
G. J. Jan*, Jenny Chen, C. H. Kuo, T. F. Lin, K. T. Pan, Glory Lin, and K. T. Hsu \\ Synchrotron Radiation Research Center, Hsinchu 30077, Taiwan \\ *Departmant of Electrical Engineering, National Taiwan University, Taipei 10767, Taiwan
}

\begin{abstract}
The prototype bunch killer has been set up at SRRC recently. The system includes two subsystems, the multibunch filling pattern shaping system and single bunch killer system. Gate RF knockout approach is used to shape the filling pattern of multibunch and to obtain a well-defined gap for machine study. The gate processes synchronize with revolution frequency. Widths of the gate signal determine the empty gap of the filling pattern. Beam knockout systems with nanosecond impulse current are also implemented which used to kill the individual electron bunch with bucket addressing capability. The purity of the single bunch is improve by the nanosecond knockout system, despite the difference of the tune and tune spread of the main and parasitic bunches, that lifts the requirement of the narrow tune spread for standard single bunch purification process. The nanosecond knockout technique also applied to kill undesired bunches in the multibunch operation mode. The preliminary results are present.
\end{abstract}

\section{INTRODUCTION}

The storage ring of SRRC operates with an $499.654 \mathrm{MHz}$ RF system and has 200 bucket locations. The required filling pattern during operation are dependent on the requirement of users or machine physicists. Multibunch filling and single bunch operations are routine achievable. Even mixed mode operation [1] is supported by existing system also. Multibunch operation modes are the most use mode. Multibunch filling are play an important role for routine operation and beam physics study. Control the filling patterns are highly desirable for various applications, such as to avoid to trap ion. The gate RF knockout technique used to shape the multibunch filling pattern for desired shapes. High purity single bunch is essential for time resolves experiments. Single bunch purity is several percentages after the injection. Since the tune spread of betatron side band are several $\mathrm{KHz}$, it is difficult to kill spurious bunches by using standard resonant excitation of transverse oscillation purification procedure $[1,2,3]$ without make loss of the main bunch at this stage. A novel method that is using impulse generator operates at betatron frequency to purify the single bunch and to kill undesired bunches at multibunch mode also demonstrated.

\section{GATE RF KNOCKOUT TECHNIQUES}

Gate RF knockout approach is used to shape the filling pattern of multibunch store mode and to obtain a well-define gap for various applications. The gate process synchronized with revolution frequency. Width of the gate signal determined the length of the empty gap of the filling pattern.

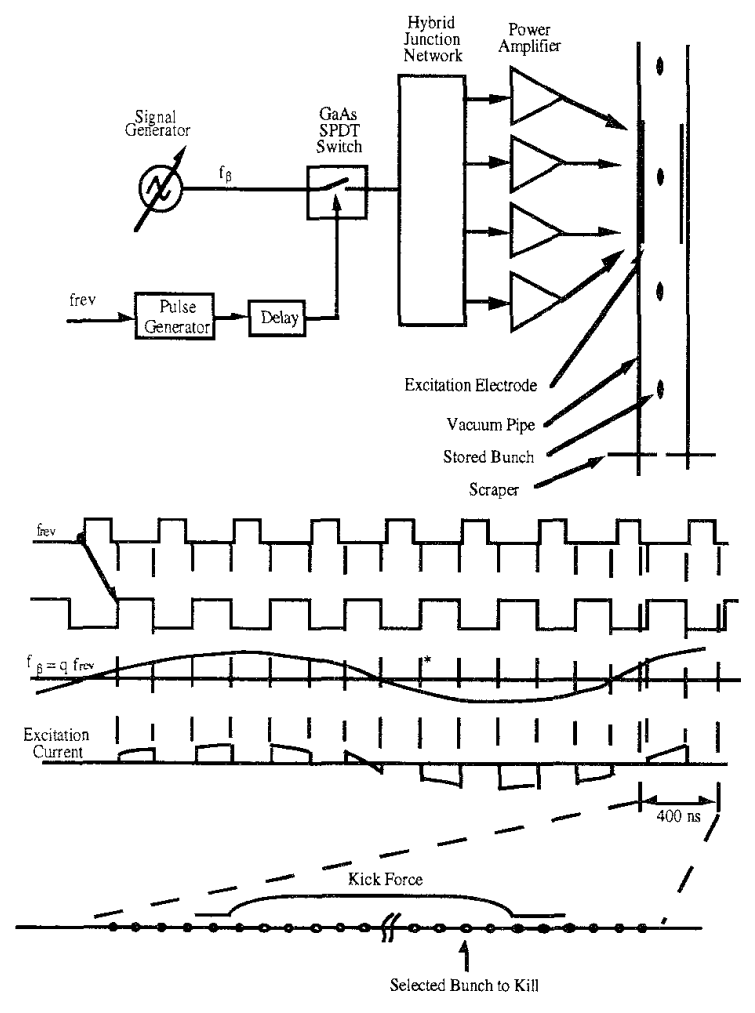

Figure 1. Multibunch filling pattern shaper

Figure 1 shown the functional block diagram of the multibunch filling pattern shaper. The revolution clock frev is used as reference of the bucket location. The delay unit determines the location of the filling bunches to be kill. The pulse generated by the pulse generator determine the length of bunch train will be kill. Betatron resonance frequency generated by a separate signal generator. The output of signal generator is gate by a GaAs RF switch with $5 \mathrm{nsec}$ rise time. The frequency ranges of the power amplifier are form $10 \mathrm{kHz}$ to $220 \mathrm{MHz}$ with rise time less than $10 \mathrm{nsec}$. Hence the shaped multibunch will be a rising and falling edge within one to three buckets.

\section{NANOSECOND BUNCH KILLER}

Beam knockout system with nanosecond impulse current is also implemented which kill the individual electron bunch at specified location. The purity of the single bunch improved by the nanosecond knockout system, despite the difference of the tune and tune spread of the main and parasitic bunches, that lifts the requirement of the narrow tune spread for standard 
single bunch purification process. The nanosecond knockout technique is also used to kill undesired bunches in the multibunch operation mode.

The nanosecond bunch killer is shown as figure 2 . The gate circuit that is coincident revolution clock and betatron oscillation frequency $f \beta$. The coincident output is delay by a delay generator. The time delay of the delay generator used to address specific bucket to kill. The output of delay trigger signal trigs four sets of impulse generator, two with positive output and two with negative output. The pulse width of the impulse generator is less than $1 \mathrm{nsec}$, and provide $100 \mathrm{~V}$ impulse to $50 \mathrm{ohm}$ load. Four excitation electrodes drive by the output of the impulse generators to exert impulse force to selected bunch.
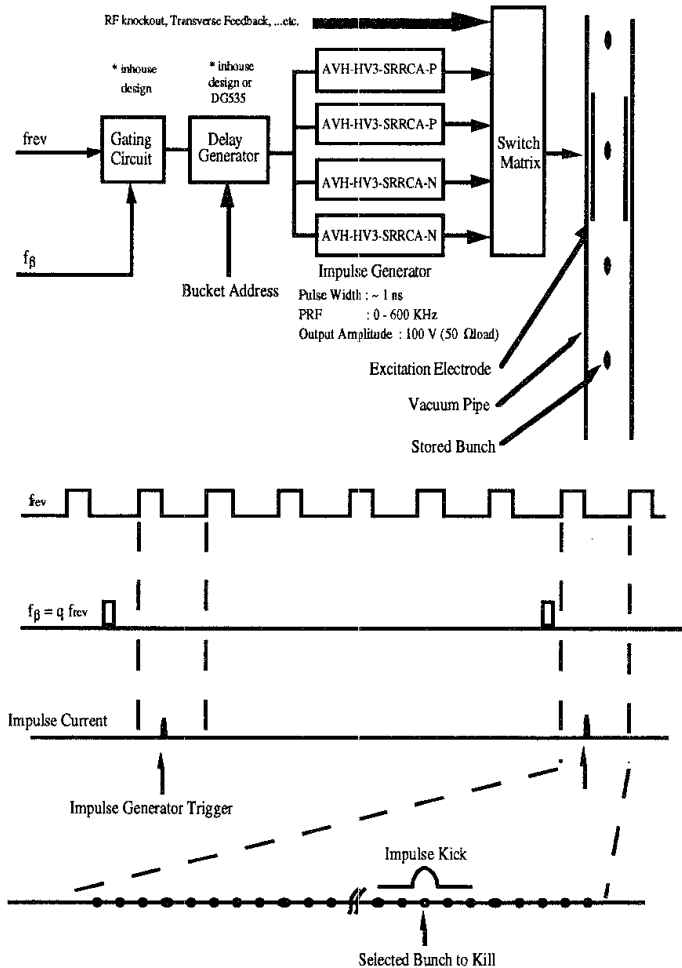

Figure 2. Nanosecond bunch killer

\section{MULTIBUNCH FILLING PATTERN SHAPING}

Multibunch filling pattern is reshaped by multibunch bunch killer to desired shape. Figure 3(a) is a designed filling pattern of the storage ring for this study. Edge of both ends cut by bunch killer are shown at figure 3(b). Reduce total beam intensity is the disadvantage of the approach.

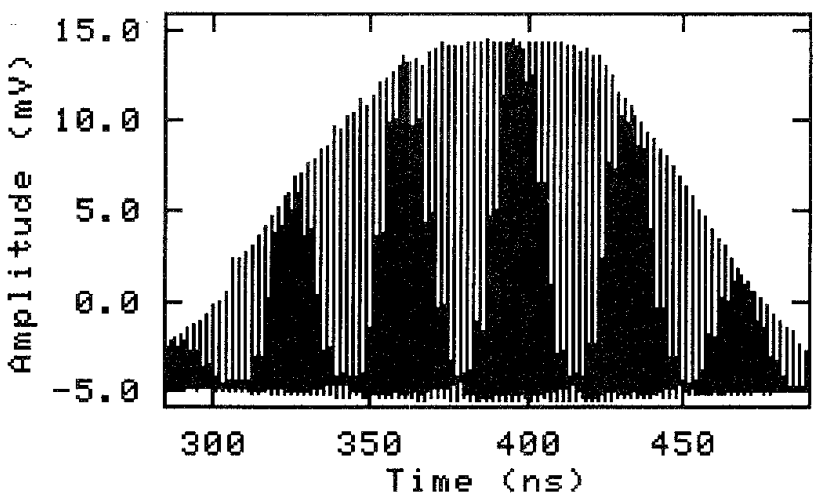

(a) Before shaping

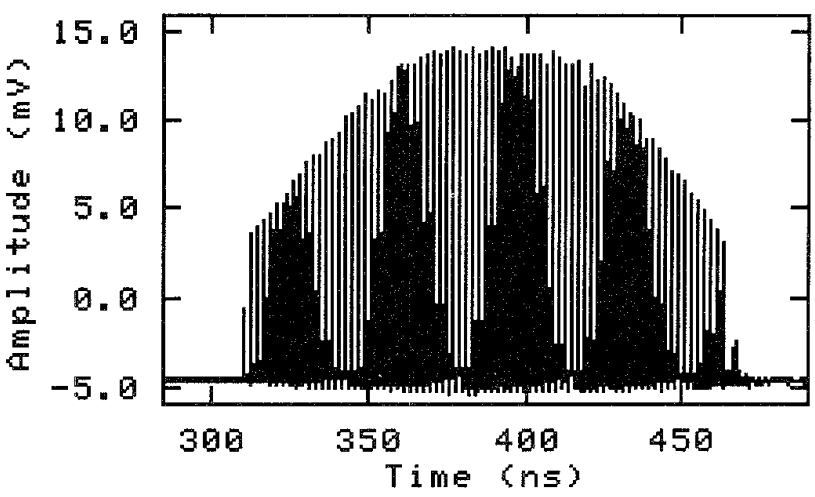

(b) after shaping

Figure 3. Multibunch filling shaping by bunch killer

\section{SINGLE BUNCH PURIFICATION}

High single bunch purity is necessary for time resolved experiments. Single bunch impurity of the storage ring is dependent upon several issues. The injector system can provide a few percentage impurities of single bunch routinely. Single bunch impurity is mainly cause by the timing jitters of the electron gun electronics. Single bunch purity can also deterioration due to various electron loss mechanisms [4]. Single bunch impurity is improved by RF knockout techniques, which based upon different betatron frequency of different bunch with different bunch current. When tune spread is too large, this technique is difficult to kill parasitic bunches. Knockout technique by using nanosecond impulse is an alternate to clean satellite bunches.

Figure 4 (a) is a typical stored single bunch with several percentage residues bunches. At the tail of the main bunches, there are some ringing signal due to electronic system. The residues bunch before main bunch are very prominent. Nanosecond bunch killer was used to kill those bunches as shown Figure 4(b), the enlargement of the detail shown at figure 4(c). Due to limited dynamic range of the observation by oscilloscope, the single bunch impurity estimated less than $10 \mathrm{e}-3$ after purification. To measure very small amount of single bunch impurity, photon counting for single bunch purity measurement is on the way. 


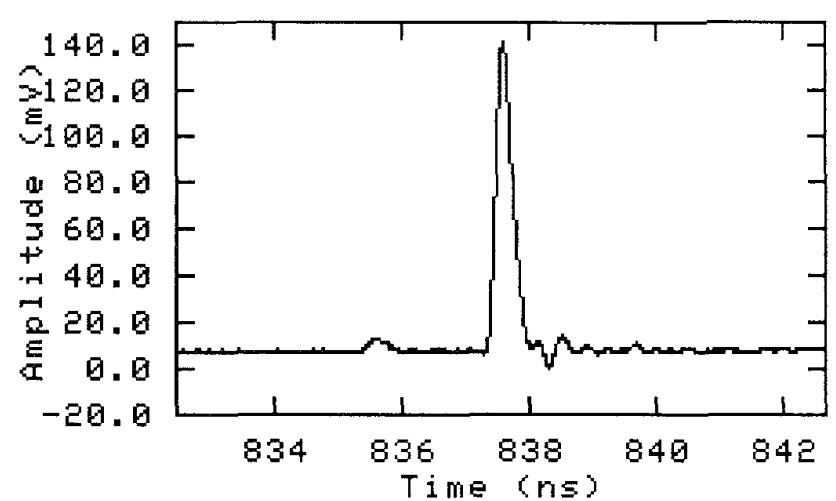

(a) Typical bunch before purify

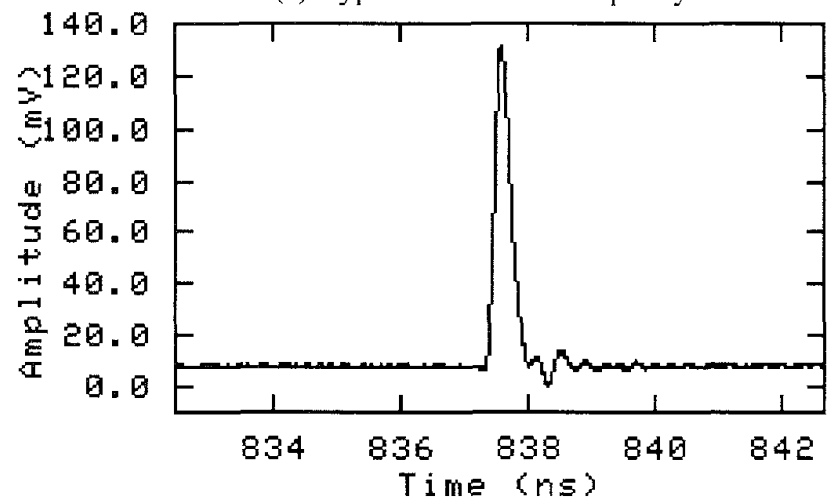

(b) Single bunch after purify

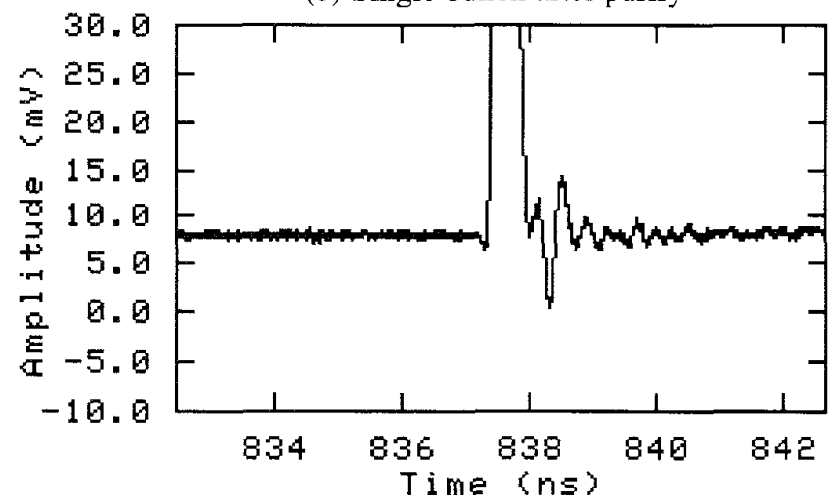

(c) Enlarge of figure (b)

Figure 4. Single bunch purification by using nanosecond bunch killer

\section{NANOSECOND BUNCH KILLER FOR MULTIBUNCH APPLICATION}

Nanosecond bunch killer was used to kill specific bunch at multibunch filling mode shown in figure 6. On the figure, six bunches are removed from a consecutive bunch at multibunch operation mode.

Randomized of multibunch filling pattern by nanosecond bunch killer is possible. Nanosecond bunch killer allows arbitrary sequence of bunches to be killed. This would allow pseudo-random sequences to be generated, affording the opportunity to add sophisticated system identification techniques to the machine analysis toolkit. Using nanosecond bunch killer to randomize the filling pattern of multibunch operation mode are more efficient then using single bunch injection and with bucket addressing to random the filling pattern of the storage ring.

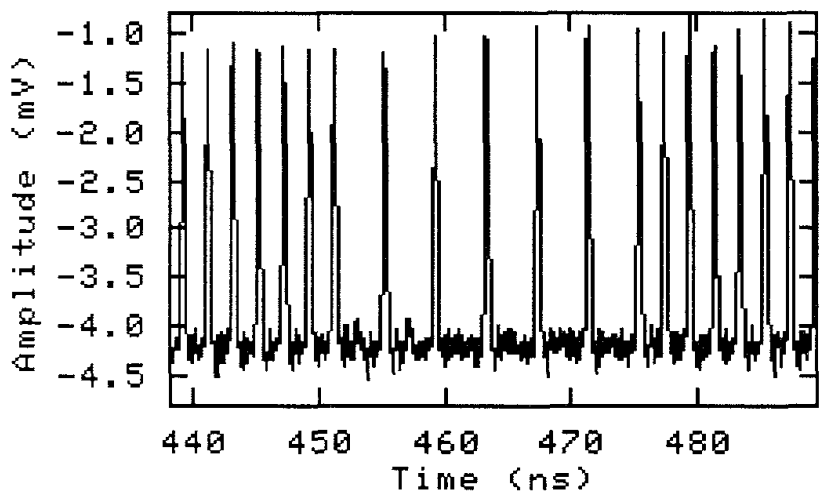

Figure 5. Multibunch filling - selected kill by nanosecond bunch killer

\section{ACKNOWLEDGMENTS}

The authors would like to thank the staff of SRRC, especially Dr. K. K. Lin and Dr. T. S. Ueng for their support running the single bunch mode and support various instruments for this study. The encourage form Prof. Y. C. Liu and Dr. Bill Weng are also highly appreciate. The advice by Dr. Jameson is also valuable for this study.

\section{REFERENCES}

[1] J. L. Revol, et al,, "Diagnostics and equipments for single bunch operation at ESRF", The Fourth European Particle Accelerator Conference, 1506 (1994).

[2] J. L. Revol, E. Plouviez, "The ESRF timing system and single bunch operation", Synchrotron Radiation News, Vol. 7, No. 4, 23 (1994).

[3] R. J. Nawrocky, et al., " A bunch killer for the NSLS Xray electron storage ring", IEEE Proceeding of Particle Accelerator Conference 1993, 2145 (1993).

[4] T. Obina, et al., "Measurement of the longitudinal bunch structure in the Photon Factory position storage ring with a photon counting system", Nucl. Instrum. and Methods A 354, 204 (1995). 